\section{$\checkmark$ VA Network Futility Guidelines: A Resource for Decisions About Withholding and Withdrawing Treatment}

\section{DOROTHY RASINSKI GREGORY}

In 1993, the Department of Veterans Affairs, through the Acting Undersecretary for Health, reorganized its VA Central Office Bioethics Committee to better serve the goals of the Veterans Health Administration and provide an accessible resource for ethics expertise. Members were recruited from a variety of offices in the Veterans Health Administration, reflecting a wide cross section of activities at the VA Central Office, and others from the field, representing different professions and geographic regions, to bring a diversity of outlook and experience. They included physicians and nurses, as well as representatives from social work and chaplain services, education, administration, geriatrics, and the VA General Counsel. Staff assistance is provided by the VA's National Center for Clinical Ethics, White River Junction, Vermont.

The committee developed several subcommittees tasked to work on topics of special interest, each of which would produce a white paper, policy recommendation, or written report. The committee as a whole, and its subcommittees, meet semiannually in Washington, DC, and more frequently, as needed, by telephone conference call and FAX.

The subcommittee assigned to consider the issue of futility prepared and submitted a white paper, entitled "Futility Guidelines: A Resource for Decisions
About Withholding and Withdrawing Treatment." It was approved by the entire committee and signed off by the Acting Undersecretary for Health, Department of Veterans Affairs. Although not defining official VA policy, the document is meant to be an educational tool, resource, or guideline for the development of local policies within VA facilities throughout the country, compatible with their patient population mix, their nature, complexity, mission, community, educational affiliation, and staff culture. It was introduced at a nationwide teleconference, transmitted to all VA facilities, in September 1994, with a follow-up teleconference scheduled in February 1995.

After a brief background describing the debate about the meaning of futility and the various factors that have fueled the concern about this issue, the white paper goes on to describe the several definitions of futility, including quantitative, qualitative, and economic, that have appeared in the bioethics literature within the past few years. It suggests that futile treatment may "be defined as that which affords no benefit, or marginal benefit, weighing the intrusiveness, burden, and risk against the ultimate outcome."

In view of the fact that the term futility has appeared to generate such passion and because it usually arises in a context of withholding or withdrawing a particular diagnostic or treatment modality, the paper recommends that the term futility itself not be defined, but rather that particular clinical situations where diagnostic or therapeutic modalities will be appropriately withheld or withdrawn, be specified. They include: 
A. Under the right to refuse treatment, where:

1) a competent patient refuses, after having received relevant information;

2) an incompetent patient's surrogate refuses, in compliance with a valid durable power of attorney for healthcare; or

3) an incompetent patient's surrogate refuses in compliance with the patient's wishes (substituted judgment) or best interest after weighing burdens and benefits.

B. Under a medical determination of futility, where:

1) treatment would only serve to prolong the dying process and bring no relief of the patient's suffering (death is inevitable and imminent and treatment includes artificial feeding and hydration where the patient is only being maintained in his/ her current state with no hope of improvement);

2) treatment would only maintain persistent vegetative state once that diagnosis had been made and its irreversibility confirmed;

3) continued treatment is in violation of an established medical center policy (see under Guiding Principles below);

4) the patient would never leave the Intensive Care Unit, for the rest of his/her life;

5) there is clear and convincing data to indicate the lack of successful outcome, for example, APACHE scores, multisystem (three or more) failure in an elderly patient, CPR in a patient with multisystem disease, etc.;

6) treatment provides only physiologic effect, but no benefit; or

7) where treatment offers no re- alistic, reasonable expectation that the physician's medical goals and the patient's personal goals and values can be realized (requires awareness of one another's goals and concurrence).

Under subheading B1 above, there is a requirement of communication between the physician and the patient's surrogate, and in the remaining six items, communication with and concurrence of the patient/surrogate is required.

A series of Guiding Principles has been included, which can be summarized as follows:

1) Under no circumstances will pain relief or such care as to maintain the patient's comfort and dignity be withheld or withdrawn.

2) Decisions about futility or the withholding or withdrawal of treatment should never be made by the attending physician alone, but only with the advice and consultation of the treating team, staff and/or consulting physicians, an appropriate formally constituted multidisciplinary committee, or in accord with established policies in the local community.

3) An existing ethics committee, or a subcommittee thereof, may be used to consider and define such instances in order to provide consensus, to assist physicians, patients, and surrogates in making these decisions. Such a committee could also be involved in "dispute resolution" where consensus is difficult between members of the treating team, patient, family, and/or surrogate. Without such a mechanism for the 
development of consensus, physicians might make ad hoc decisions, overly influenced by individual bias. In addition, the application of institutional consensus may protect the patient from burdensome measures. Care that will not be provided should include that which is outside the limits of professional standards, that which is negligent, and that which compromises the physician's integrity. Resource allocation and rationing decisions are separate healthcare issues and, if cost is to be a factor in withholding or withdrawing treatment, it should only be as the result of an established institutional or national policy, and not determined on an ad hoc basis by a physician and/or administrator.

The white paper concludes with a set of immediate goals and items of continuing emphasis, including encouraging the use of advance directives and the Durable Power of Attorney for Health Care, and early and frequent communication between physician and patient/surrogate; increased use of "time-limited" trials to allow room for compromise with reevaluation of the clinical status; the increased use of ethics committees as sounding boards to mediate, guide, and offer support; and increased patient and professional education with patient empowerment. Long-range goals include more outcomes research; development of treatment guidelines and explicit policies based on such research, to become prevailing medical practice, with a "feedback loop" for modification and amendment where appropriate; awareness of the concepts of limitations and fairness; evolution and development of societal consensus regarding which treatments are not appropriate to offer or provide under particular circumstances; and the use of facility multidisciplinary committees, with involvement of patients/families/surrogates, to review generic cases, to help define criteria or explicit facility policies regarding which treatments or procedures should be considered futile in specific clinical situations and should therefore not be instituted or should appropriately be discontinued.

Again, it should be noted that this document is not official VA policy, but is meant only to be a resource or guideline to offer assistance and a basis for thought and critical discussion among VA hospital staffs, ethics committees, and members of administration.

From a personal perspective, I can only applaud the development of such committee efforts as white papers and guiding principles. They demonstrate that a large bureaucracy can move forward and deal with the cutting-edge issues and difficult problems that arise daily in clinical practice, rather than follow clumsily and adopt a program only after it has become an accepted widespread standard throughout the community.

One might also hope that the development and distribution of such white papers will inspire and motivate hospital staffs and communities, in the VA and in the private sector, to define what care should be considered futile, and therefore not to be provided, similar to efforts instituted in Denver. ${ }^{1}$ The VA should not be considered an entity unto itself, either in its individual facilities or as a Department of the Federal Government, but rather as an integral part of the nation's healthcare system and a critical element in its delivery.

\section{Notes}

1. Murphy, Fonucane. Archives of Internal Medicine 1993;153:1641. 